\title{
Disialoganglioside GD3 is released by microglia and induces oligodendrocyte apoptosis
}

\author{
BM Simon ${ }^{1,2,5}$, F Malisan ${ }^{3,5}$, R Testi ${ }^{3}$, P Nicotera $^{1,2}$ and \\ M Leist ${ }^{\star, 1,4}$ \\ ${ }^{1}$ Department of Molecular Toxicology, University of Konstanz, 78457 Konstanz, \\ Germany \\ ${ }^{2}$ MRC Toxicology Unit, Leicester, UK \\ 3 Department of Experimental Medicine and Biochemical Sciences, University of \\ Rome 'Tor Vergata', 00133 Rome, Italy \\ ${ }^{4}$ H. Lundbeck A/S, 2500 Valby, Denmark \\ 5 The first two authors contributed equally to this study \\ * Corresponding author: M Leist, Neurobiology, H. Lundbeck A/S, Ottiliavej 9 , \\ 2500 Valby, Denmark. Tel: +45-3630-1311; Fax: +45-3644-0043; \\ E-mail: male@lundbeck.com
}

Received 8.11.01; revised 22.1.02; accepted 22.1.02

Edited by M Piacentini

\begin{abstract}
Increased brain ganglioside levels are a hallmark of various neuroinflammatory pathologies. Here, we provide evidence that murine microglia can secrete disialoganglioside GD3 upon exposure to inflammatory stimuli. Comparison of different neural cell types revealed a particular and specific sensitivity of oligodendrocytes towards exogenous GD3. Oligodendrocyte death triggered by GD3 was preceded by degeneration of cellular processes, and associated with typical features of apoptosis, such as chromatin condensation, exposure of phosphatidylserine, release of cytochrome $c$ from mitochondria, and loss of mitochondrial membrane potential, followed by the loss of plasma membrane integrity and detachment of disintegrated oligodendrocytes. Overexpression of $\mathrm{bcl}-2$ partially protected oligodendrocytes from death. In contrast, treatment with the pan-caspase inhibitor zVAD-fmk did not prevent phosphatidylserine exposure, chromatin margination at the nuclear periphery, and death, although caspase- 3 was blocked. Thus, GD3 produced by microglia under neuroinflammatory conditions may function as a novel mediator triggering mitochondria-mediated, but caspase-independent, apoptosis-like death of oligodendrocytes.

Cell Death and Differentiation (2002) 9, 758-767. doi:10.1038/ sj.cdd. 4401027
\end{abstract}

Keywords: oligodendrocyte; microglia; apoptosis; ganglioside; mitochondria; demyelination

Abbreviations: CSF, cerebrospinal fluid; CGC, cerebellar granule neurons; CNPase, cyclic nucleotide phosphodiesterase; cyt $c$, cytochrome $c$; EH-1, ethidium homodimer-1; FCS, foetal calf serum; GD3, disialoganglioside 3; GD1a, disialoganglioside 1a; GD1b, disialoganglioside 1b; GM3, monosialoganglioside 3; LPS, lipopo- lysaccharide; MOG, myelin oligodendrocyte glycoprotein; MPT, mitochondrial permeability transition; MTT, 3-(4,5-Dimethyldiazol2-yl)-3,5-diphenyltetrazolium bromide; MS, Multiple Sclerosis; NO, nitric oxide; PS, phosphatidylserine; P/S; penicillin/streptomycine; TLC, thin layer chromatography; TMRE, tetramethylrhodamineethylester; TNF $\alpha$, tumour necrosis factor alpha; $\Delta \Psi_{\mathrm{m}}$, mitochondrial membrane potential; zVAD-fmk, N-benzyloxycarbonyl-Val-Alaaspartyl-fluoromethylketone

\section{Introduction}

GD3 is a disialoganglioside that can function in the intracellular signalling cascade leading to leukocyte apoptosis. The ganglioside causes opening of the mitochondrial permeability transition pore complex and the release of apoptogenic factors, both in cells and on isolated mitochondria. ${ }^{1-4}$ Intracellular accumulation of GD3 is observed in some models after triggering the death receptor CD95. Inhibitors of GD3 synthesis protect from apoptosis under such circumstances. ${ }^{5,6}$ Since the addition of exogenous GD3 induces apoptosis, 5,7 GD3 might also take a role as intercellular mediator of death.

In the healthy brain of adult rodents, GD3 is found only in early oligodendrocyte precursors, microglia, and some defined neuronal cell types. ${ }^{8-11}$ In pathologic conditions, ganglioside composition and distribution in the brain changes. For instance, microglia increase their GD3 content upon activation, ${ }^{12}$ and elevated cerebrospinal fluid (CSF) GD3 levels have been reported in Multiple Sclerosis (MS) and leukoaraiosis. ${ }^{13,14}$ Moreover, the GD3 content is increased in MS plaques in comparison to healthy white matter, while GM1, the most abundant ganglioside in normal brain, decreases. ${ }^{15}$ So far, neither the source of gangliosides in CSF nor a potential direct cytotoxic action of GD3 to cells of the central nervous system has been investigated.

The cells most heavily affected by neuroinflammation are oligodendrocytes. A large body of evidence suggests that cytokines, such as interferon- $\gamma$ and members of the tumour necrosis factor death ligand family (TNF/CD95L/NGF), are responsible for their degeneration. ${ }^{16-19}$ Since myelin damage also occurs in various cytokine knockout mice, and data on the toxicity of cytokines on oligodendrocytes are controversial, it is likely that additional mediators contribute to oligodendrocyte degeneration. ${ }^{20-23}$ These might include nitric oxide (NO)/peroxynitrite, ${ }^{24,25}$ glutamate $^{26}$ and a direct attack of myelin by macrophages. ${ }^{27}$

Preliminary data on GD3 toxicity in oligodendrocytes was already included in a report focusing on the total chemical synthesis of this ganglioside. ${ }^{28}$ In this study, we examined GD3 as a new potentially cytotoxic mediator synthesized and secreted by brain macrophages under inflammatory conditions. We showed that microglia cells, stimulated with 
lipopolysaccharide (LPS) or bacteria, release GD3 into the culture medium. Subsequently, we investigated in detail whether and how GD3 would trigger cell death in oligodendrocytes. Specificity of GD3 mediated toxicity was defined by examination of cell type selectivity and by comparison to structurally similar gangliosides. The mode of cell death was analyzed on the basis of morphological changes of nucleus and cytoplasm, and the differential role of caspase-3 for chromatin condensation was put under scrutiny.

\section{Results}

\section{GD3 secretion by microglia cells in vitro}

We investigated first whether activated brain macrophages might release GD3, since elevated levels of gangliosides have been found in demyelinating diseases and neuroinflammatory conditions. Primary murine microglia were exposed either to LPS or inactivated $E$. coli bacteria for up to $72 \mathrm{~h}$. Cell culture supernatants were analyzed for their GD3 content by thin layer chromatography (TLC).

In supernatants from control cells, GD3 concentrations were below the detection limit at all time points analyzed. In contrast, supernatants from stimulated cells were GD3positive at $24 \mathrm{~h}$ with the higher concentration of E.coli. The GD3-content increased over the following $48 \mathrm{~h}$ also after stimulation with LPS or the lower concentration of E.coli (Figure 1). Thus, activated microglia can produce significant increases of extracellular GD3, which in vivo might accumulate in the interstitial space.

\section{Selective oligodendrocyte toxicity triggered by GD3}

The above data show that microglia increase GD3 production upon activation. Therefore, we evaluated the sensitivity of different brain cells to extracellularly added GD3. Neither microglia nor astrocytes were significantly affected by $400 \mu \mathrm{M}$ of this ganglioside (Figure $2 \mathrm{~A}$ ). Also, in primary neurons (cerebellar granule cells) more than $90 \%$ of the cells survived exposure to $400 \mu \mathrm{M}$ GD3. In contrast, primary oligodendrocytes showed a remarkable sensitivity towards GD3: more than $70 \%$ of these cells died within $24 \mathrm{~h}$. This manifested in retraction of cellular processes and breakdown of the

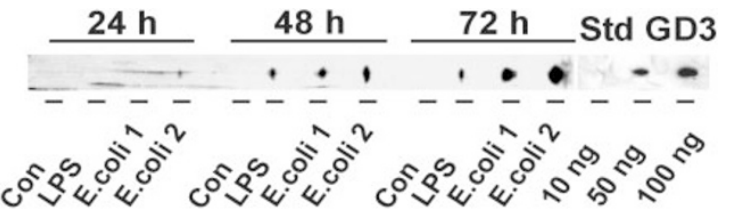

Figure 1 GD3 production by microglia. Murine microglia cells were stimulated either with solvent (con), $10 \mu \mathrm{g} / \mathrm{ml}$ LPS (LPS), $0.8 \mu \mathrm{g} / \mathrm{ml}$ of $E$. coli (1) or $4 \mu \mathrm{g} / \mathrm{ml}$ of $E$. coli (2) for the indicated times. For quantification of GD3 in cell culture supernatants, aliquots of the medium were extracted with chloroform/methanol and separated by TLC. Subsequently, GD3 was immunostained with specific antibodies. The amounts recovered from the culture medium were around the detection limit at $24 \mathrm{~h}\left(25 \mathrm{pmol} / 10^{6} \mathrm{cells}\right)$ and steadily increased to around $900 \mathrm{pmol} / 10^{6}$ cells at $72 \mathrm{~h}$. One complete data set out of three similar experiments is shown membrane network (Figure 2B: $6 \mathrm{~h}, 12 \mathrm{~h}$ ). In the late phase of degeneration, cells detached from the substrate (Figure 2B: $24 \mathrm{~h})$.

\section{Specific susceptibility of oligodendrocytes to GD3}

The selective susceptibility of oligodendrocytes to GD3 was put under scrutiny in three different ways. First, it was examined whether oligodendrocytes are generally more sensitive to exogenous gangliosides than other cells. For this purpose they were incubated with gangliosides metabolically (GM3) or structurally (GD1a, GD1b) related to GD3. None of the other gangliosides induced the pronounced toxicity that was observed with GD3 (Figure 2C).

Second, we used chemically synthesised GD3 to compare its effects with the bovine brain-derived GD3. ${ }^{28}$ Synthetic and natural GD3 induced the same extent of toxicity in primary oligodendrocytes (Figure 2C). However, higher concentrations of synthetic GD3 had to be used

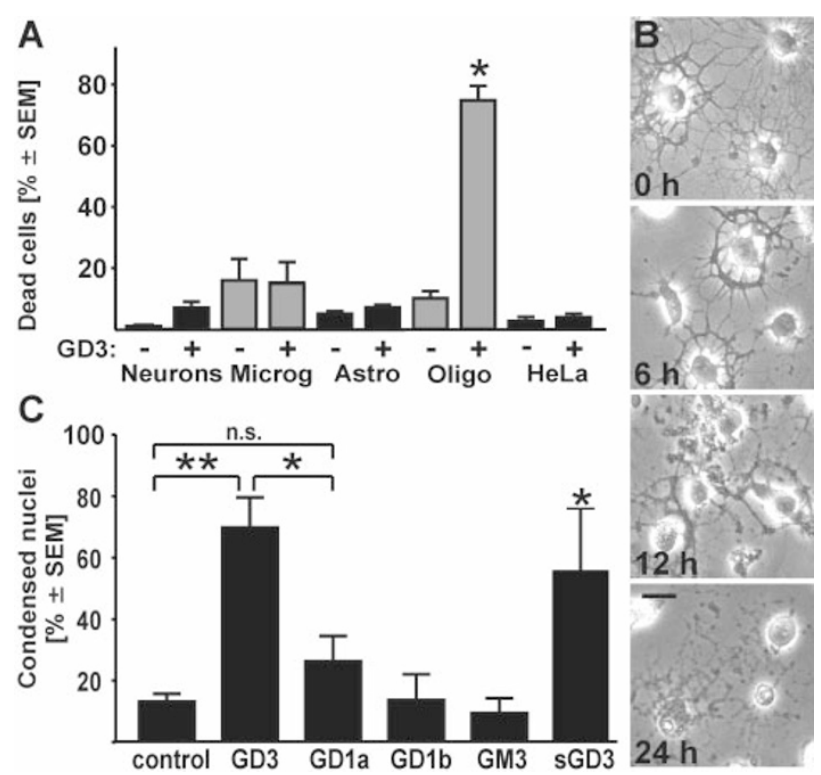

Figure 2 Specificity and selectivity of GD3-mediated oligodendrocyte toxicity. (A) Cultures of cerebellar granule neurons (CGC), microglia, astrocytes, HeLa cells or oligodendrocytes were incubated for $24 \mathrm{~h}$ with $400 \mu \mathrm{M}$ GD3. Oligodendrocyte death was evaluated from cultures co-stained with $\mathrm{H}-33342$ and anti-CNPase. CNPase-positive cells with nuclear changes were scored as dead oligodendrocytes. Cell death in CGC, microglia, astrocytes and HeLa cells was evaluated after staining with the two chromatin dyes Sytox ${ }^{\mathbb{R}}(1 \mu \mathrm{M})$ and $\mathrm{H}-33342(1 \mu \mathrm{g} / \mathrm{ml})$, and cells with permeabilised plasma membranes and/or with condensed nuclei were scored as dead. Data are means +S.E.M. from at least three experiments (HeLa cells two experiments) with evaluation of at least six scoring fields per experiment. Student's $t$-test: ${ }^{\star} P<0.01$. (B) Oligodendrocytes were exposed to $300 \mu \mathrm{M}$ GD3 for $24 \mathrm{~h}$. A sequence of retraction and fragmentation of membrane processes, followed by detachment from the culture surface was obvious beginning at $6 \mathrm{~h}$ after GD3 addition. At $12-24 \mathrm{~h}$ of incubation cells were heavily damaged and detached easily. Scale bar: $20 \mu \mathrm{m}$. (C) Oligodendrocytes were incubated for $24 \mathrm{~h}$ with $400 \mu \mathrm{M}$ of GD3, GD1a, GD1b, GM3 or $800 \mu \mathrm{M}$ synthetic GD3 (sGD3), respectively. Then, cells were stained for CNPase and condensed nuclei in CNPase-positive cells were scored as described above. Data from five experiments were subjected to one-way ANOVA followed by Tukey's multiple comparison test. Control vs GD3: $P<0.001$, control vs GD1a: $P>0.05$ (n.s.), GD3 vs GD1a: $P<0.01$, control vs sGD3: $P<0.05$ 
$(800 \mathrm{M})$ to obtain oligodendrocyte damage equal to that seen with bovine brain-derived GD3. In addition, the possibility of ceramide contamination in the commercially obtained natural GD3 was investigated by combined gas chromatography/mass spectrometry. No ceramides were detected (data not shown).

Third, the sensitivity of our standard oligodendrocyte preparation (4-days-old) was compared to oligodendrocyte cultures of different age and different cell lines. The concentration response curves of the nuclear changes induced by GD3 (from 100 to $400 \mu \mathrm{M}$ ) in 2-day-old (less than $20 \%$ fully differentiated oligodendrocytes) and 6-dayold (more than $75 \%$ fully differentiated oligodendrocytes) oligodendrocytes were not different from the curve obtained with 4-day-old cells (data not shown). Thus, the differentiation state of oligodendrocytes did not significantly affect their sensitivity. On the other hand, neither HeLa cervix carcinoma cells (Figure 2A), nor WEHI-S fibrosarcoma cells (not shown) were sensitive to GD3 under our culture conditions. Also $\mathrm{O} 4 \mathrm{Cbneu}$, an immortalised cell line descending from 04 -positive oligodendrocyte progenitors ${ }^{29}$ were not damaged by GD3 (data not shown).

\section{Nuclear apoptotic features of GD3-mediated oligodendrocyte death}

As shown above, changes in oligodendrocyte morphology became evident by phase contrast microscopy at $6-12 \mathrm{~h}$ after GD3 exposure (Figure 2B) and developed in parallel with the redistribution of chromatin. The ganglioside triggered a defined sequence of changes in nuclear morphology. While chromatin became clumpy in the early stages, pronounced condensation at the nuclear periphery and fragmentation to individual masses occurred during later stages of the cell damage (Figure 3A). GD3 toxicity towards oligodendrocytes was assessed by scoring cells with both types of nuclear alterations. Changes in chromatin structure were induced by GD3 in a concentration dependent manner (Figure 3B).

As damaged oligodendrocytes tend to detach from the culture substrate, we quantified oligodendrocyte degeneration in long-term experiments by scoring the total cell number in a dish and calculating the amount of lost cells as percentage of control. Experiments of longer duration (48 h) revealed, that oligodendrocytes degenerated also after exposure to only $100 \mu \mathrm{M}$ GD3 (Figure 3C). In an experiment with $72 \mathrm{~h}$ exposure, even $50 \mu \mathrm{M}$ GD3 caused more than $60 \%$ of all oligodendrocytes to detach from the substrate. Further experiments were performed at GD3 concentrations of $300 \mu \mathrm{M}$. Under these conditions about $40 \%$ of the oligodendrocytes were lost from the coverslip within $24 \mathrm{~h}$

\section{Phosphatidylserine-exposure during GD3-mediated oligodendrocyte apoptosis}

By staining living GD3-treated oligodendrocytes with annexin$\checkmark$ we observed the translocation of phosphatidylserine (PS) to the outer leaflet of the cell membrane (Figure 4A). Cell membrane integrity was controlled by co-incubating cells with

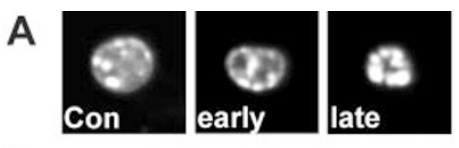

B
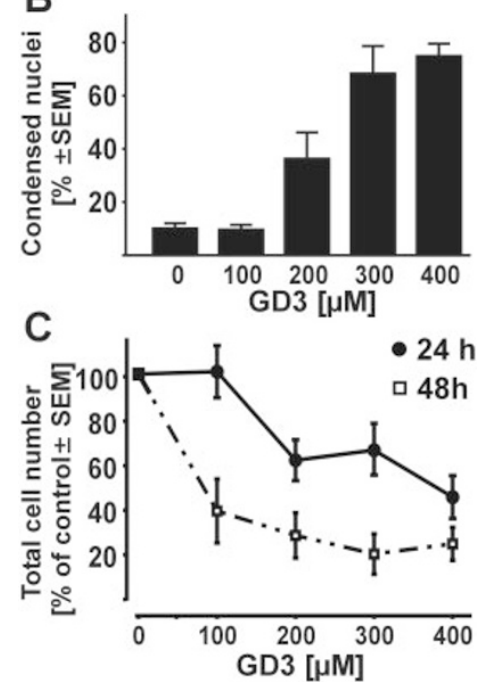

Figure 3 Nuclear morphology changes induced by GD3 in oligodendrocytes. (A) Oligodendrocytes were treated with GD3 $(300 \mu \mathrm{M})$ and representative examples of nuclear morphology are shown. Compared to normal nuclei (con) chromatin showed first peripheral hyperchromasy (early) and then more severe condensation and fragmentation (late). Image size: $20 \mu \mathrm{m} \times 20 \mu \mathrm{m}$. (B) For quantification of the concentration-dependent GD3 toxicity, condensed nuclei of CNPase positive oligodendrocytes were scored after chromatin staining with $\mathrm{H}-33342$. (C) Cells were incubated with GD3 for the indicated times, fixed and stained for CNPase. The scores of oligodendrocytes remaining attached to the culture dish are given as percentage of untreated controls of the same cell preparation. Data are means \pm S.E.M. from 3-6 cell preparations

ethidium-homodimer-1 (EH-1), which stains only nuclei of cells with disrupted cell membrane. Notably, untreated control cells often stained positive for PS in the periphery of membrane processes, but never on their cell bodies. After GD3 stimulation, PS-translocation to the cell surface appeared to proceed from the periphery to the soma, until the whole cell surface was annexin- $V$ positive (Figure $4 \mathrm{~A}$, $12 \mathrm{~h})$.

Quantification of PS-exposure with relation to nuclear changes showed that the membrane lipid alterations paralleled the nuclear processes (Figure 4B). Eventually, cell lysis took place several hours later $(12-18 \mathrm{~h}$ after GD3 addition) as indicated by staining of nuclei by $\mathrm{EH}-1$ (Figure 4B).

\section{Mitochondrial changes associated with GD3-induced apoptosis}

As GD3 has been shown before to directly affect mitochondria, ${ }^{1-3}$ we investigated the fate of these organelles in GD3treated oligodendrocytes (Figure 4A). Cells were stained with tetramethylrhodamine-ethylester (TMRE), a dye sensitive for the changes in mitochondrial membrane potential $\left(\Delta \Psi_{\mathrm{m}}\right)$. Control cells showed the typical staining pattern of mitochondria. The specificity of the staining was shown by rapid and 
complete loss of fluorescence after exposure to the mitochondrial uncoupler carbonyl-cyanide-4-trifluoro-methoxyphenylhydrazone (FCCP, not shown). Subtle changes in mitochondrial morphology (shortening) preceded the loss of fluorescence intensity (data not shown). Then, staining intensity decreased, and finally was completely lost, indicating loss of $\Delta \Psi_{\mathrm{m}}$ (Figure 4, TMRE $12 \mathrm{~h}$ ). A quantification of this observation at the population level shows that loss of $\Delta \Psi_{\mathrm{m}}$
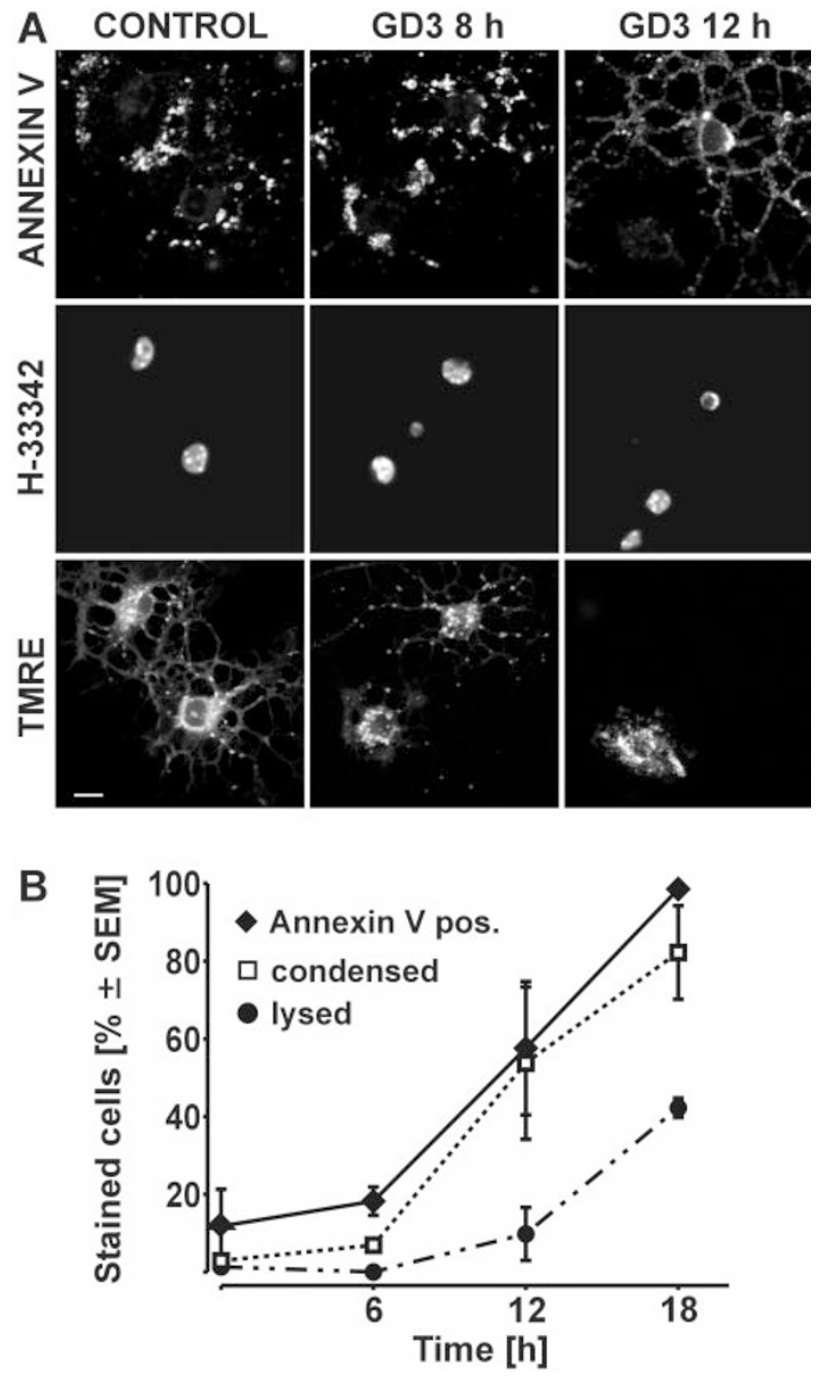

Figure 4 Phosphatidylserine exposure and loss of mitochondrial membrane potential induced by GD3. Oligodendrocytes were exposed to GD3 $(300 \mu \mathrm{M})$ and analyzed at different time points. (A) Cells were incubated for $15 \mathrm{~min}$ with a mixture of $\mathrm{H}-33342(1 \mu \mathrm{g} / \mathrm{ml}$, chromatin), $\mathrm{EH}-1(0.5 \mu \mathrm{M}$, chromatin, cell membrane intactness) and the $\Delta \Psi_{\mathrm{m}}$ sensitive dye TMRE $(20 \mathrm{nM}$, mitochondrial functional status). Then they were stained with fluoresceine-labelled annexin-V and analyzed quickly in cold buffer by multi-channel confocal microscopy. The individual fluorescent channels recorded are shown separately for a representative group of cells. Note the annexin-V-staining at the tip of the myelin extension of control cells and the condensation of chromatin and loss of TMRE-staining in the annexin-V-positive cell (12 h). Only cells that were negative for $\mathrm{EH}-1$ staining were imaged. Scale bar: $10 \mu \mathrm{m}$. (B) Oligodendrocytes were scored for annexin V-staining on the cell body, for nuclear staining by EH-1 (cell membrane lysis), and for condensed chromatin (condensed). Data are means \pm S.E.M. from data obtained from three independent cell preparations had started at $6 \mathrm{~h}$ after GD3-exposure. By $12 \mathrm{~h}$ up to $70 \%$ of the oligodendrocytes had completely lost TMRE staining (Figure 5A).

We studied then, whether mitochondrial functional damage coincided with redistribution of cyt $c$. Release of cyt $c$ from mitochondria into the cytosol was examined on the single-cell level in relation to other cell death parameters (Figure 6). Generally, the release of cyt $c$ coincided with first changes in chromatin structure. Comparison of parallel experiments revealed that cyt $c$ translocation to the cytosol preceded the loss of $\Delta \Psi_{\mathrm{m}}$ (Figure 5A).

GD3-toxicity in oligodendrocytes might be linked to the induction of mitochondrial permeability transition, and be modulated by $\mathrm{Bcl}-2$ expression levels as described in other cell types before. ${ }^{4}$ To test the role of $\mathrm{Bcl}-2$, we prepared primary cultures from animals on $\mathrm{C} 57 \mathrm{BL} / 6$ background overexpressing a human transgene for $b c l-2$, and from nontransgenic littermates. Oligodendrocytes were incubated with $100-300 \mu \mathrm{M}$ of GD3 for $24 \mathrm{~h}$ and analyzed by scoring of nuclear morphological changes. The toxicity of $300 \mu \mathrm{M}$ GD3 was reduced by about $60 \%$ in $b c /-2$ transgenic cells (Figure 5B). Statistical analysis of the concentration-
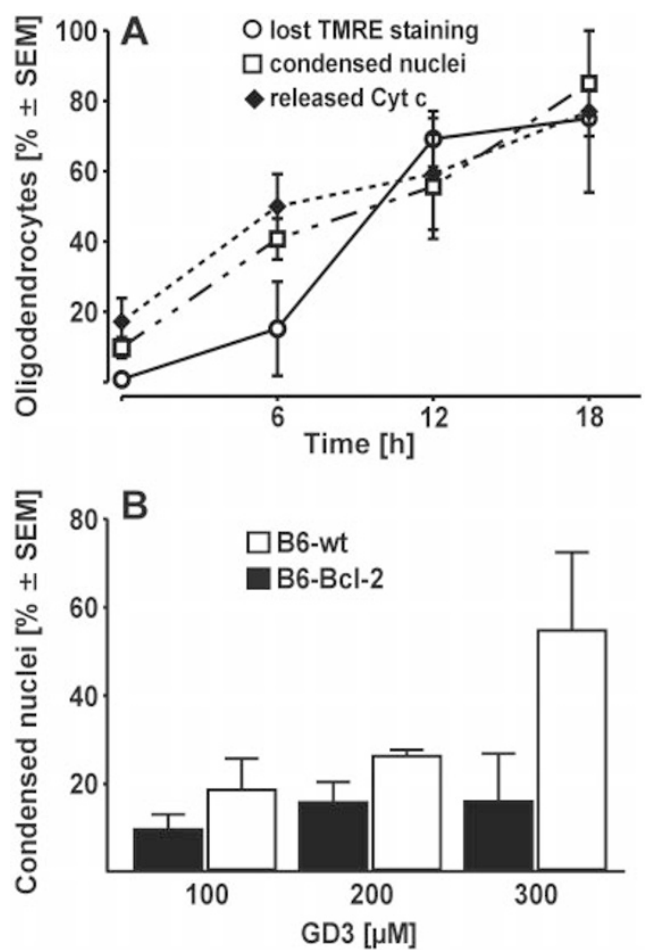

Figure 5 Role of mitochondria in GD3-induced oligodendrocyte apoptosis. Cells were incubated with GD3 $(300 \mu \mathrm{M})$ for the indicated times and fixed. (A) CNPase positive cells with a delocalised cyt $c$ stain (as in Figure $6 \mathrm{~A}$ ) or with condensed chromatin were scored. Data are means \pm S.E.M. from five cell preparations. In parallel non-fixed cultures, the percentage of oligodendrocytes (oligodendrocyte-morphology) with lost TMRE staining was counted. (B) Oligodendrocyte cultures ( $n=3$ each) were prepared from C57BL/ 6 mice (B6wt, white bars) or from mice expressing a human bcl-2 transgene (B6-Bcl-2, black bars). Cells were stimulated with the indicated GD3 concentrations for $18 \mathrm{~h}$, fixed and stained for CNPase and chromatin. Condensed nuclei were counted, and two-way ANOVA was performed with respect to genotype and GD3 concentration: $P<0.001$ for the effect of the genotype 
dependent effects of GD3 by two-way ANOVA confirmed a highly significant difference between the two genotypes $(P \leqslant 0.001)$.

\section{Activation and differential role of caspases during GD3-triggered apoptotic processes in oligodendrocytes}

Since classical apoptotic features, as observed in GD3triggered oligodendrocyte death, are often associated with caspase-activation, ${ }^{30,31}$ we investigated whether any of the cell death features observed here depend on the activation of these proteases. First, we studied whether caspases in the apoptosome pathway (downstream of cytochrome $c$ release) were activated at all. Using an immunocytochemical approach, we stained oligodendrocytes for the presence of active/cleaved caspase-3. We obtained clear evidence for caspase- 3 activation in all cells that had released cyt $c$ from mitochondria and displayed fully condensed chromatin. This approach allowed us to test whether the polycaspase-inhibitor $\mathrm{N}$-benzyloxycarbonyl-Val-Ala-aspartyl-fluoromethylketone (zVAD-fmk), which is stable in cell culture medium for $\geqslant$

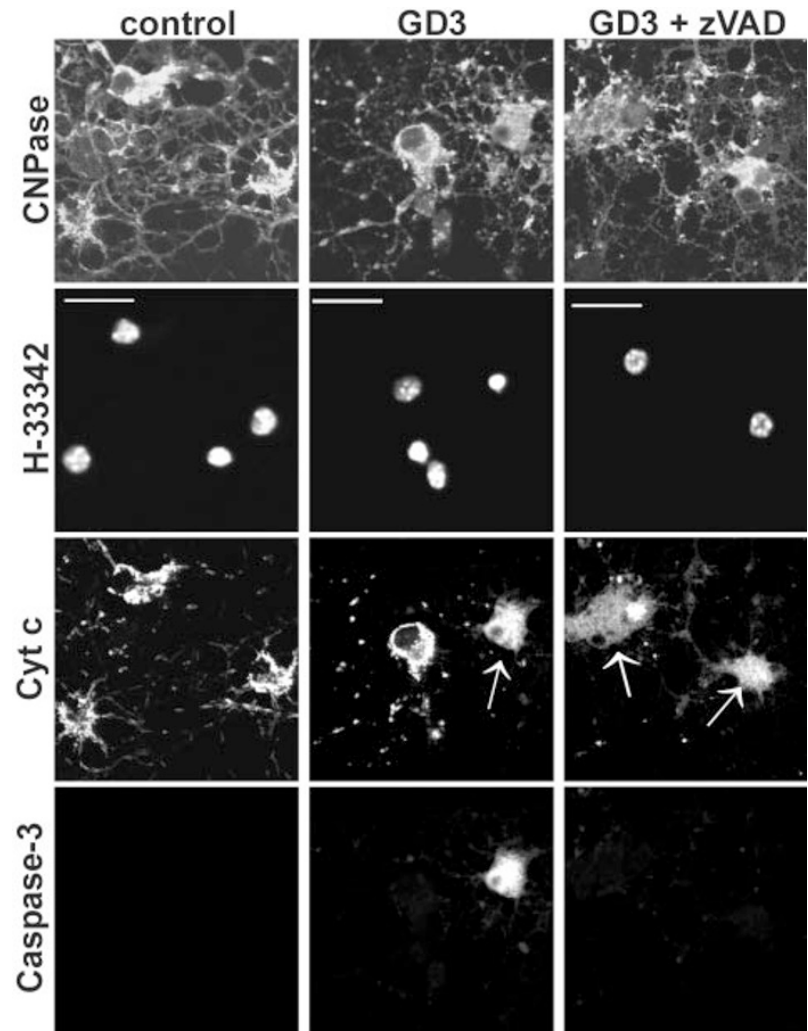

Figure 6 GD3 induced cytochrome $c$ release and caspase-3 activation. Oligodendrocyte cultures were exposed to combinations of GD3 $(300 \mu \mathrm{M})$ and zVAD-fmk $(100 \mu \mathrm{M})$ for $12 \mathrm{~h}$ and then fixed. They were simultaneously stained for CNPase to identify oligodendrocytes, for cyt $c$, for active caspase-3 and for DNA (H-33342). Fluorescence was recorded with a confocal scanning microscope. Control cells show the typical distribution of cyt $c$ in mitochondria while GD3-exposed cells treated with or without zVAD-fmk show release of cyt $c$ (arrows). Activation of caspase- 3 is evident in GD3 treated cells with condensed chromatin and released cyt $c$. ZVAD-fmk-treated cultures show cyt $c$ release, but neither active caspase- 3 nor full chromatin condensation. Scale bar: $20 \mu \mathrm{m}$
$24 \mathrm{~h}^{32}$ entered oligodendrocytes and inhibited caspase processing. ZVAD-fmk completely prevented GD3-induced caspase-3-processing in oligodendrocytes (Figure 6).

In contrast, GD3-induced cyt $c$ delocalisation from mitochondria to the cytosol was not prevented by zVADfmk preincubation (Figure 6, arrows). Clear evidence was also found that $z V A D$-fmk inhibited neither phosphatidylserine exposure (data not shown) nor the extent of membrane lysis (60\% after $16 \mathrm{~h}$ with or without zVAD-fmk).

However, when nuclear alterations were examined, pronounced effects of zVAD-fmk treatment became evident. The typical apoptotic chromatin condensation changed to an apoptosis-like morphology of marginated, clumpy chromatin aggregation (Figure 7A). Full apoptotic chromatin condensation was almost entirely prevented by the caspase-inhibitor (Figure 7B).

\section{Discussion}

GD3-triggered apoptosis has been examined in a variety of cell types, but it has remained unknown whether this ganglioside might affect neural cells. Here we provide evidence that GD3 is produced and secreted by activated microglia and that it exerts selective and potent toxic effects on oligodendrocytes. Taken together, these findings suggest the possibility that the reported elevated levels of GD3 in tissue and CSF of MS patients ${ }^{13,15}$ are not epiphenomena, but might actively contribute to the pathologic mechanism in demyelinating diseases.

\section{Gangliosides and CNS injury}

Under pathologic conditions like white matter degeneration, HIV-caused neurodegeneration or medulloblastoma, GD3

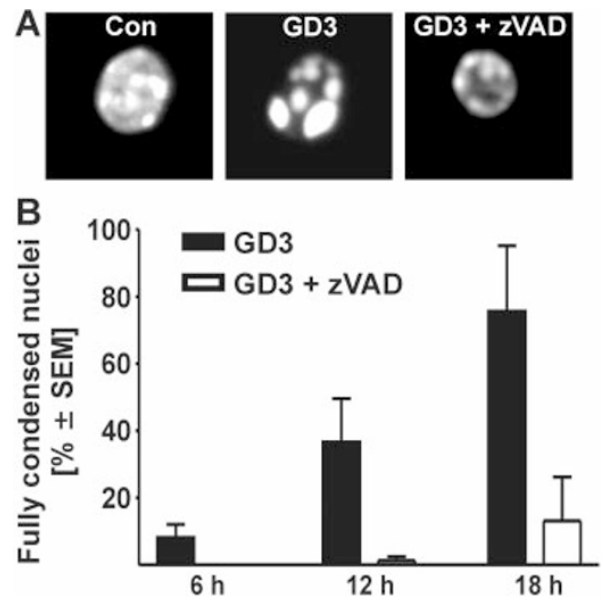

Figure 7 Caspase inhibition does not prevent oligodendrocyte toxicity but changes nuclear condensation. Oligodendrocytes were exposed to GD3 and zVAD-fmk and immunostained as in Figure 5. (A) Representative nuclei show that zVAD-fmk stopped chromatin condensation at the early stage of peripheral hyperchromasy. Image size: $20 \mu \mathrm{m} \times 20 \mu \mathrm{m}$. (B) The number of nuclei with fully condensed and fragmented chromatin was scored at different times of incubation with $300 \mu \mathrm{M}$ GD3. Data are means \pm S.E.M. from three experiments. Two-way ANOVA yielded $P<0.001$ for the effect of $Z$ VAD-fmk on chromatin condensation over time 
and other gangliosides accumulate in CSF. ${ }^{12-14,33}$ On the basis of their role for cell viability at least two major classes of gangliosides seem to exist. One shows neuroprotective effects $^{34-36}$ and is exemplified by the monosialoganglioside GM1. The other class is represented by GD3, which constitutes $3-8 \%$ of all gangliosides in adult brain. ${ }^{37}$ This disialoganglioside is a specific inducer of apoptosis in leukocytes, hepatocytes and various cell lines., ${ }^{4,7,38} \mathrm{We}$ provide evidence here that oligodendrocytes are particularly susceptible to the cell death-inducing capacity of this lipid.

\section{Which is the source of extracellular GD3?}

The question arises what the sources of pathologically elevated ganglioside levels might be. One possibility is lymphocytes that infiltrate the CNS in autoimmune disease and undergo apoptosis in large numbers. ${ }^{39}$ In fact, lymphocytic cells produce GD3 during apoptosis. ${ }^{5,40}$ Secondly, degenerating neural cells or myelin structures might release GD3 upon their disintegration. A hint to a third option comes from the observation that microglia accumulate GD3 as reaction to brain injury. ${ }^{12,41}$ Thus, activated microglia might produce and possibly secrete the ganglioside. In fact, our data show, that microglia cells are capable of releasing GD3 in substantial amounts when incubated with the strong proinflammatory stimuli LPS or inactivated bacteria. An increased sensitivity in GD3 detection will be needed to investigate the activity of cytokines in inducing GD3 release. Preliminary results (not shown) indeed indicate that IFN $\gamma$ either together with a low amount of LPS or with TNF $\alpha$ was able to stimulate GD3 release. Activation of microglia occurs during various CNS pathologies, and especially accumulated and clustered microglia might constitute a source of substantially raised extracellular GD3 levels.

\section{The concentration-dependence of GD3 effects}

We observed significant oligodendrocyte toxicity in a concentration range of $50-300 \mu \mathrm{M}$ GD3, which corresponds to the amounts of GD3 used with other cells. ${ }^{4,5,7}$ However, these concentrations are clearly above the approximate $10 \mu \mathrm{M}$ required to trigger permeability transition in isolated mitochondria. $^{1,4,7}$ This can be explained by the physico-chemical properties of GD3 that support the formation of micelles in aqueous solution. At the beginning of the experiment, a metastable 'solution' of GD3 can be produced by vigorous sonication. Micelle formation and growth hardly plays a role for experiments with isolated mitochondria, where effects are measured within minutes. However, in such experiments GD3 was considerably less effective when it was not sonicated immediately prior to use (own unpublished observation). In long-term cell culture experiments, free GD3 is lost from the medium to micelles. The kinetics of this process is determined by the homogeneity of GD3 itself. Synthetic GD3 (uniform C18 fatty acid residues) forms micelles much more efficiently than natural GD3 (lipid chains with different length). Thus, synthetic GD3 had to be used here in higher concentrations than bovine brain-derived GD3 to obtain equal biological potency.

The biologic action and its concentration dependence of lipids like ceramide or GD3 further depend on their cellular localization. Both can be present in cell membranes and take signalling functions, but also trigger protease activation and apoptosis, e.g. when they are targeted to mitochondria $^{1,3,4,7}$ or lysosomes. ${ }^{42}$ The pathways of uptake and distribution of GD3 in cells warrant further investigations, since they may shed light on the reasons for different susceptibility of diverse cell types.

\section{Specificity of GD3-mediated oligodendrocyte toxicity}

Assuming an involvement of GD3 in oligodendrocyte degeneration during inflammatory demyelination, we analyzed the response of different brain cells to GD3-exposure. GD3 did not prove to be generally cytotoxic. Rather, it acted with high selectivity on oligodendrocytes compared to other neural cell types and selected cell lines. While astrocytes and microglia remained resistant to high concentrations, cerebellar granule neurons (CGC) showed some sensitivity to GD3. But also when concentrations were raised to $600 \mu \mathrm{M}$, only a small percentage of neurons degenerated after exposure to GD3 $(30 \%$ cell death within $24 \mathrm{~h}$ in CGC exposed to $600 \mu \mathrm{M}$, vs $>70 \%$ in oligodendrocytes exposed to $400 \mu \mathrm{M})$.

With lipids used at micro-molar concentrations, there is a danger of chemical impurity or biologically unspecific effects. However, we used GD3 from various suppliers and sources (milk and brain), excluded ceramide contamination by chemical analysis and used a chemically synthesized molecule to obtain pure and homogeneous material. ${ }^{28}$ The resistance of oligodendrocytes to chemically similar gangliosides excluded unspecific effects.

\section{Mechanisms of GD3-mediated oligodendrocyte toxicity}

Most of what is known on GD3-mediated programmed cell death centres around the ganglioside's effects on mitochondria. Studies in isolated mitochondria provide proof of a direct induction of mitochondrial permeability transition (MPT) by GD3. ${ }^{1-3,7}$ However, the situation in cells might be more complex, and MPT might be a secondary effect of other GD3 actions. ${ }^{3}$ Other cell-death modulating effects of GD3 might be linked to its ability to block the translocation of the antiapoptotic transcription factor NF- $\kappa$ b to the nucleus. ${ }^{38}$ Our observations of cytochrome $c$ release to the cytoplasm and loss of $\Delta \Psi_{\mathrm{m}}$ indicate a central role of mitochondria in GD3induced oligodendrocyte death. The protective effect of Bcl-2 supports a causal role of mitochondria in GD3 induced oligodendrocyte degeneration, and is in concordance with previous observations. $^{4}$

As described for many other apoptosis models, we found that the release of cyt $c$ from mitochondria triggered the activation of caspase-3. Closer examination of this process revealed that caspase inhibition by $z V A D$-fmk affected the morphology of dying cells, but not the extent of cell death. Similar observations were made earlier in GD3-exposed U937 cells. ${ }^{3}$ In a search for alternative execution pathways we tested a range of other protease inhibitors (DEVD$\mathrm{CHO}$, YVAD-cmk, calpain inhibitors I, II, III, a selective cathepsin B inhibitor, TLCK, E64). None of them affected 
GD3 toxicity. However, other molecules that can potentially cause nuclear alterations in a caspase independent mode include $\mathrm{AIF}^{43}$ or endonuclease $\mathrm{G}^{44}$

\section{The mode of cell death triggered by GD3}

GD3 triggered oligodendrocyte apoptosis, as evidenced by the particular type of chromatin condensation, translocation of PS, and activation of caspase-3. It has become clear in the last years that death commitment in apoptosis is frequently linked to mitochondrial changes and that caspases activated downstream mainly determine the shape or kinetics of death. ${ }^{30,31,45}$ The break of the outer mitochondrial membrane leading to the release of cytochrome $c$ also triggers redistribution of other intermembrane proteins. For instance, translocation of AIF as a consequence of GD3-exposure and its control by $\mathrm{Bcl}-2$ have been observed previously. ${ }^{4} \mathrm{AIF}$ alone is able to trigger caspase-independent programmed cell death, and this pathway becomes particularly evident when caspases are inhibited. ${ }^{43}$ In this case, chromatin condensation is less compact and lumpier than under caspase-3 control, ${ }^{31,43}$ exactly as observed in the present study, when caspases were inhibited by zVAD-fmk. Caspase inhibition can occur in pathological situations as a consequence of oxidative stress or ATP depletion. ${ }^{46,47}$ Accordingly, a significant amount of degenerating oligodendrocytes in MS lesions does not show classical apoptotic features. ${ }^{48,49}$

The specific susceptibility of oligodendrocytes to GD3 released from microglia under inflammatory conditions might explain a number of aspects of oligodendrocyte degeneration: myelin damage that precedes the actual cell loss; cell death that is in part independent of caspases; dying cells with mixed non-apoptotic and apoptosis-like chromatin and an important role of inflammatory mediators. This study contributes to the discussion about the involvement of lipid molecules in pathologies of the central nervous system, and direct research efforts to new targets with potential use for treatment of demyelinating disease.

\section{Materials and Methods}

\section{Materials}

Cell culture media were from Gibco BRL Life Technologies (Eggenstein, Germany). Gangliosides and other chemicals were from Sigma (Deisenhofen, Germany). Chemically synthesized GD3 was a kind gift of Dr. J Castro-Palomino. ${ }^{28} \mathrm{EH}-1$, Sytox ${ }^{\circledR}$, TMRE, secondary antibodies coupled with Alexa dyes and a fluorescent protein labelling kit were obtained from Molecular Probes (Eugene, OR, USA). Some primary antibodies were from Pharmingen (Hamburg, Germany). Hoechst-33342 ( $\mathrm{H}-33342)$ and annexin-V were obtained from Roche Biochemicals (Mannheim, Germany).

\section{Animals}

Primary cultures were prepared from specific pathogen free BALB/C mice provided by the animal unit of the University of Konstanz, Germany. Transgenic animals expressing the human bcl-2-gene under the control of an ubiquitin promoter ${ }^{50}$ were generously provided by Dr. J Schenkel (Heidelberg, Germany). Breeding was performed by crossing transgenic males with wt (C57BL/6) females. All experiments were performed in accordance with international guidelines to minimise pain and discomfort (NIH-guidelines and European Community Council Directive 86/609/EEC).

\section{Cell culture}

Mouse oligodendrocytes were isolated from a primary mixed brain cell culture $^{51}$ prepared from BALB/c embryos at days $15-16$ of gestation. After removal of the meninges, cortex and cerebellum were incubated for $3 \mathrm{~min}$ in $1 \%$ trypsin. Digestion was stopped by addition of $10 \%$ foetal calf serum (FCS) and the cells were dissociated by triturating with fire polished Pasteur pipettes. The cell suspension was plated on poly-L-lysine coated $(100 \mu \mathrm{g} / \mathrm{ml})$ culture flasks in Dulbecco's Modified Eagle Medium (DMEM) with $50 \mathrm{U} / \mathrm{ml}$ penicillin, $50 \mu \mathrm{g} / \mathrm{ml}$ streptomycin (P/S) and 10\% FCS. After 14 to 20 days first microglia cells were detached from the primary culture, shaking gently by hand. In a second step, after strong shaking of the flasks, the fraction of cells enriched in oligodendrocyte precursors was harvested. To further the depletion of microglia, the suspension was incubated for $45 \mathrm{~min}$ on a petri dish. Non-adherent cells were plated (secondary culture) on polyL-lysine coated coverslips $\left(7 \times 10^{4}\right.$ cells/coverslip). After $2 \mathrm{~h}$, medium was exchanged for DMEM supplemented according to Sato ${ }^{52}, 1 \%$ FCS, $10 \mathrm{ng} / \mathrm{ml}$ biotin and $100 \mu \mathrm{g} / \mathrm{ml}$ bovine serum albumine. Fifty per cent of this medium had been conditioned by astrocytes for $24 \mathrm{~h}$. Cells were in most of the cases used 4 days after preparation of the secondary culture. Maturation of oligodendrocytes was monitored by staining for antigens specific for differentiation stages of oligodendrocytes (O4-antigen, O1-antigen, Cyclic Nucleotide Phosphodiesterase (CNPase), Myelin Oligodendrocyte Glycoprotein, listed in sequence of appearance during differentiation). ${ }^{53,54}$ After 4 days in vitro about $30 \%$ of the cells stained positive for CNPase. Microglia cells were obtained as $95 \%$ pure culture from the first shaking step (see oligodendrocyte preparation). They were seeded on plastic culture dishes and maintained in DMEM plus $1 \%$ FCS and P/S. LPS $(10 \mu \mathrm{g} / \mathrm{ml})$, as well as inactivated Escherichia coli (E. coli, 0.8 and $4 \mu \mathrm{g} / \mathrm{ml}$ ), triggered an inflammatory response in microglia characterised by release of TNF $\alpha$ and production of NO (data not shown). Viability of microglia was controlled at the end of the experiment within the same cultures used for GD3 production. Unimpaired capacity of the cells to reduce MTT (3-(4,5-Dimethyldiazol-2-yl)-3,5-diphenyltetrazolium bromide) showed that neither stimulated nor control microglia cells had an impaired viability after an experimental period of $72 \mathrm{~h}$ (data not shown). GD3 itself was not proinflammatory, since it neither triggered TNF $\alpha$-release nor NO-production (data not shown).

Astrocytes were prepared by trypsinising $(0.25 \%$ trypsin) the feeder layer of the primary mixed cell culture and replating cells on poly-L-lysine coated plastic dishes. Astrocytes were cultured in DMEM with $1 \% \mathrm{FCS}$ and P/S.

Murine cerebellar granule neurons were cultivated as described before. $^{55}$

O4CBneu ${ }^{29}$ cells were cultivated as described. HeLa (ATCC no. HTB-22) and WEHI-S ${ }^{56}$ were cultivated in DMEM with $10 \%$ FCS and $\mathrm{P} / \mathrm{S}$.

To prepare oligodendrocyte cultures from bcl-2-overexpressing animals, cortex and cerebellum were cleaned from meninges and maintained for $24 \mathrm{~h}$ in hibernation medium $\left(\mathrm{KCl} 30 \mathrm{mM}, \mathrm{MgCl}_{2}\right.$ $0.24 \mathrm{mM}, \mathrm{NaH}_{2} \mathrm{PO}_{4} 10.95 \mathrm{mM}, \mathrm{Na}_{2} \mathrm{HPO}_{4} 5 \mathrm{mM}$, Glucose $5 \mathrm{mM}$, $\mathrm{pH}$ 7.2, sorbitol ad $295 \mathrm{mOsm}$ ) while genotyping was carried out according to an existing protocol. ${ }^{57}$ Genotypes were determined by PCR amplification of a $460 \mathrm{bp}$ fragment of the human bcl-2-gene using the primers ( $5^{\prime}$ to $\left.3^{\prime}\right)$ act tgt ggc tca gat agg cac cca g and $\left(5^{\prime}\right.$ to $\left.3^{\prime}\right)$ cga 
ctt cgc cga gat gtc cag cca g. Brains from transgenic or wild-type embryos were pooled respectively and processed as usual. To control bcl-2-expression on protein level, astrocyte proteins were analyzed by Western blot $^{57}$ using a monoclonal anti-hu-Bcl-2 antibody (clone Bcl2-100, Sigma).

\section{Immunostaining}

Cells were fixed in $4 \%$ paraformaldehyde for $10 \mathrm{~min}$ at room temperature (RT), permeabilised with $0.1 \%$ Triton $X-100$ for $10 \mathrm{~min}$ and incubated with a monoclonal CNPase antibody (clone 11-5B, Sigma), a monoclonal cytochrome $c$ antibody (clone 6H2.B4, Pharmingen) or a monoclonal rabbit active-caspase-3 antibody (clone C92-605, Pharmingen). Primary antibodies were detected with a goatanti-mouse or goat-anti-rabbit antibody coupled to Alexa-488 or Alexa568. DNA was stained with $\mathrm{H}-33342(0.5 \mu \mathrm{g} / \mathrm{ml})$ and coverslips were mounted in Aquapolymount (Polysciences, Warrington, PA, USA). To perform a simultaneous four-colour staining for cytochrome $c$ (cyt $c$ ), CNPase, active caspase- 3 and chromatin, reagents were applied in the following sequence: anti-active-caspase-3 antibody overnight, $4^{\circ} \mathrm{C}$, anti-cytochrome $c$ antibody for $30 \mathrm{~min} \mathrm{RT}$, a mixture of goat-antirabbit Alexa-488 and goat-anti-mouse cy5 (Jackson Immuno Research, West Grove, PA, USA) 30 min RT, anti-CNPase coupled directly to Alexa-568 and $\mathrm{H}-33342$. Cells were analysed with a confocal laser-scanning microscope (TCS-4D confocal scanning system, Leica AG, Benzheim and Leica Lasertechnik, Heidelberg, Germany) equipped with a UV-laser and a visible-light laser.

\section{Scoring of oligodendrocyte damage}

To quantify the oligodendrocyte detachment after GD3 exposure, total number of oligodendrocytes (CNPase-positive cells) was counted in 10 randomly chosen fields of $310 \times 310 \mu \mathrm{m}$ and normalized to the number of untreated cells. In further experiments CNPase-positive cells with nuclei stained by $\mathrm{H}-33342$ were scored for changes in nuclear structure. For each condition, nine fields of $310 \times 310 \mu \mathrm{m}$ were counted. To monitor cyt $c$ redistribution in apoptosis, oligodendrocytes were co-stained for cyt $c$ and CNPase. CNPase-positive cells were scored for diffuse distribution of cyt $c$ in the cell.

\section{Viability assays in other cells}

Toxicity in CGCs, astrocytes, microglia and other cells was assessed after staining living cells with a mixture $0.5 \mu \mathrm{M}$ SYtox ${ }^{\circledR}$ (nonmembrane permeable, green-fluorescent chromatin stain) and $1 \mu \mathrm{g} /$ $\mathrm{ml} \mathrm{H}-33342$ (cell permeable, blue-fluorescent chromatin stain) as described. ${ }^{58}$ Cells with lysed membrane or condensed nuclei were counted as dead.

\section{Visualization of phosphatidylserine translocation}

Surface PS-expression was analyzed by annexin-V staining and confocal microscopy as described. ${ }^{59}$ Briefly, 10 min before the annexin- $\mathrm{V}$ staining, cells were stained in the original culture medium with $\mathrm{EH}-1(0.5 \mu \mathrm{g} / \mathrm{ml}), \mathrm{H}-33342(1 \mu \mathrm{g} / \mathrm{ml})$ and the fluorescent indicator TMRE $(20 \mathrm{nM})$ for $10 \mathrm{~min}$ to visualize plasma membrane integrity, chromatin structure and mitochondrial membrane potential $\left(\Delta \Psi_{\mathrm{m}}\right)$. Cells were incubated briefly in annexin-buffer (HEPES $10 \mathrm{mM}, \mathrm{NaCl}$ $140 \mathrm{mM}, \mathrm{CaCl}_{2} 2.5 \mathrm{mM}, \mathrm{pH} 7.4$ ) and then incubated with FITCcoupled annexin-V. Cells were analyzed with a fluorescent microscope (Leica, DM-IRB) or by confocal microscopy (Leica, TCS). To score oligodendrocytes with positive annexin- $\mathrm{V}$ staining and lost mitochon- drial membrane potential, oligodendrocytes were identified by morphology in phase contrast and counted in six fields $(300 \times 300 \mu \mathrm{m})$ for each condition.

\section{GD3 production by microglia}

To measure the GD3 production by microglia, $1.5 \times 10^{5}$ cells/well were cultivated in DMEM with $10 \%$ FCS and P/S. Microglia cells were stimulated in DMEM (1\% FCS and P/S) with LPS from Salmonella abortus equi (BioClot, Aidenbach, Germany) or with inactivated Escherichia coli (K12 strain, Molecular Probes) for 24,48 or $72 \mathrm{~h}$. Supernatant of two equally stimulated wells was pooled after this times, centrifuged for $5 \mathrm{~min}$ at $4^{\circ} \mathrm{C}$ to remove cells or cell remnants and stored at $-20^{\circ} \mathrm{C}$ until analysis of the GD3 content.

\section{Quantitation of GD3 by thin layer chromatography}

Gangliosides were extracted as previously described with minor modifications. ${ }^{5}$ Briefly, supernatant was treated for $30 \mathrm{~min}$ with trypsin $(0.5 \mathrm{mg} / \mathrm{ml})$ and EDTA $(0.2 \mathrm{mg} / \mathrm{ml})$. They were then sonicated for $30 \mathrm{~s}$ at $10 \mathrm{~W}$, and debris was removed by centrifugation for $10 \mathrm{~min}$ at $15000 \times g$. Gangliosides were extracted from supernatant with chloroform/methanol $(1: 2)$ to obtain a ratio of $1: 0.8: 1.6$ (supernatant/chloroform/methanol). After vortex and $10 \mathrm{~min}$ centrifugation at $15000 \times g$, the upper phase was recovered. Two volumes of methanol were added and evaporated to dryness under nitrogen gas. Gangliosides were then resuspended in chloroform/methanol (2:1) and loaded on a silica gel HP-TLC plate (Merck, Darmstadt, Germany) and chromatographed in chloroform $/$ methanol/ $\mathrm{CaCl}_{2} 0.2 \%(2: 1: 0.2)$. Plates were treated with $0.5 \%$ poly-iso-butyl-metacrylate in hexane and dried. To carry out immunostaining for GD3, the plate was incubated for $1 \mathrm{~h}$ with R24 monoclonal anti-GD3-antibody ${ }^{60}$, followed by an horseradish peroxidase-conjugated anti-lgG secondary antibody. Specifically stained bands were detected by chemiluminescence.

\section{Statistic}

All experiments were repeated in at least three cell preparations. If not indicated otherwise, statistical significance was calculated on the original data sets using the Student's $t$-test. When variances within the compared groups were not homogeneous, the Welch test was applied.

\section{Acknowledgements}

The excellent technical assistance of Elvira Gawlitta-Gorka is gratefully acknowledged. We are grateful to Dr. J Schenkel (Institute of Physiology II, University of Heidelberg, Germany) and Dr. R Jäger (Forschungszentrum Karlsruhe, Germany) for providing bcl-2 transgenic mice, to Dr. T Mürdter (Dr. M Fischer-Bosch-Institute for Clinical Pharmacology, Stuttgart, Germany) for measurements of ceramides by combined gas-chromatography and mass-spectroscopy and to Dr. J Castro-Palomino (Bayer AG, Wuppertal, Germany) and Prof. Dr. RR Schmidt (University of Konstanz, Germany) for chemical synthesis of GD3. This study was supported by the DFG grant We686/18, by AIRC, by ISS Progetto Sclerosi Multiple as well as the EEC grants BMH4CT97-2410 and 12029-97-06 F1ED ISP D. F Malisan was supported by a fellowship from FIRC. 


\section{References}

1. Kristal BS and Brown AM (1999) Apoptogenic ganglioside GD3 directly induces the mitochondrial permeability transition. J. Biol. Chem. 274: 23169-23175

2. Pastorino JG, Tafani M, Rothman RJ, Marcineviciute A, Hoek JB and Farber JL (1999) Functional consequences of the sustained or transient activation by Bax of the mitochondrial permeability transition pore. J. Biol. Chem. 274: $31734-$ 31739

3. Garcia-Ruiz C, Colell A, Paris R and Fernandez-Checa JC (2000) Direct interaction of GD3 ganglioside with mitochondria generates reactive oxygen species followed by mitochondrial permeability transition, cytochrome c release, and caspase activation. FASEB J. 14: 847-858

4. Rippo MR, Malisan F, Ravagnan L, Tomassini B, Condo I, Costantini P, Susin SA, Rufini A, Todaro M, Kroemer G and Testi R (2000) GD3 ganglioside directly targets mitochondria in a bcl-2-controlled fashion. FASEB J. 14: 2047-2054

5. De Maria R, Lenti L, Malisan F, d'Agostino F, Tomassini B, Zeuner A, Rippo MR and Testi R (1997) Requirement for GD3 ganglioside in CD95- and ceramideinduced apoptosis. Science 277: 1652-1655

6. De Maria R, Rippo MR, Schuchman EH and Testi R (1998) Acidic sphingomyelinase (ASM) is necessary for fas-induced GD3 ganglioside accumulation and efficient apoptosis of lymphoid cells. J. Exp. Med. 187: 897-902

7. Scorrano L, Petronilli V, Di Lisa F and Bernardi P (1999) Commitment to apoptosis by GD3 ganglioside depends on opening of the mitochondrial permeability transition pore. J. Biol. Chem. 274: 22581-22585

8. Ellison JA and de Vellis J (1995) Amoeboid microglia expressing GD3 ganglioside are concentrated in regions of oligodendrogenesis during development of the rat corpus callosum. Glia. 14: 123-132

9. Kawai K, Mori M, Watarai S and Yasuda T (1994) Immunohistochemical demonstration of ganglioside GD3 in the central nervous system. Neurosci. Res. 19: $119-124$

10. Molander $\mathrm{M}, \mathrm{Berthold} \mathrm{CH}$, Persson $\mathrm{H}$ and Fredman $\mathrm{P}(2000)$ Immunostaining of ganglioside GD1b, GD3 and GM1 in rat cerebellum: cellular layer and cell type specific associations [In Process Citation]. J. Neurosci. Res. 60: 531-542

11. Vitry S, Avellana-Adalid V, Hardy R, Lachapelle F and Baron-Van Evercooren A (1999) Mouse Oligospheres: From Pre-Progenitors to functional oligodendrocytes. J. Neurosci. Res. 58: 735-751

12. Andersson L-M, Fredman P, Lekman A, Rosengren $L$ and Gisslén $M$ (1998) Increased cerebrospinal fluid ganglioside GD3 concentrations as a marker of microglial activation in HIV type 1 infection. Aids Res. Human Retrovir. 14: $1065-1069$

13. Miyatani N, Saito M, Ariga T, Yoshino H and Yu RK (1990) Glycosphingolipids in the cerebrospinal fluid of patients with multiple sclerosis. Mol. Chem. Neuropathol. 13: 205-216

14. Tarvonen-Schröder S, Blennow K, Lekman A, Fredman $P$, Räihä I and Sourander L (1997) Gangliosides and Sulfatide in cerebrospinal fluid in leukoaraiosis. Dement. Geriatr. Cogn. Disord. 8: 174-179

15. Yu RK, Ledeen RW and Eng LF (1974) Ganglioside abnormalities in multiple sclerosis. J. Neurochem. 23: 169-174

16. Selmaj K, Raine CS, Cannella B and Brosnan CF (1991) Identification of lymphotoxin and tumor necrosis factor in multiple sclerosis lesions. J. Clin. Invest. 87: 949-954

17. D'Souza SD, Alinauskas KA and Antel JP (1996) Ciliary neurotrophic factor selectively protects human oligodendrocytes from tumor necrosis factormediated injury. J. Neurosci. Res. 43: 289-298

18. Vartanian T, Li Y, Zhao M and Stefansson K (1995) Interferon-gamma-induced oligodendrocyte cell death: implications for the pathogenesis of multiple sclerosis. Mol. Med. 1: 732-743

19. D'Souza SD, Bonetti B, Balasingam V, Cashman NR, Barker PA, Troutt AB, Raine CS and Antel JP (1996) Multiple sclerosis: Fas signaling in oligodendrocyte cell death. J. Exp. Med. 184: 2361-2370

20. DittelBN, Merchant RM and Janeway Jr CA. (1999) Evidence for Fas-dependent and Fas-independent mechanisms in the pathogenesis of experimental autoimmune encephalomyelitis. J. Immunol. 162: 6392-6400

21. Frei K, Eugster HP, Bopst M, Constantinescu CS, Lavi E and Fontana A (1997) Tumor necrosis factor alpha and lymphotoxin alpha are not required for induction of acute experimental autoimmune encephalomyelitis. J. Exp. Med. 185: 2177 2182
22. Liu J, Marino MW, Wong G, Grail D, Dunn A, Bettadapura J, Slavin AJ, Old L and Bernard CC (1998) TNF is a potent anti-inflammatory cytokine in autoimmunemediated demyelination. Nat. Med. 4: 78-83

23. Agresti C, D'Urso D and Levi G (1996) Reversible inhibitory effects of interferongamma and tumour necrosis factor-alpha on oligodendroglial lineage cell proliferation and differentiation in vitro. Eur. J. Neurosci. 8: 1106-1116

24. Mitrovic B, Parkinson J and Merrill JE (1996) An in vitro model of oligodendrocyte destruction by nitric oxide and its relevance to multiple sclerosis. Methods 10: $501-513$

25. Willenborg DO, Staykova MA and Cowden WB (1999) Our shifting understanding of the role of nitric oxide in autoimmune encephalomyelitis: a review. J. Neuroimmunol. 100: 21-35

26. Pitt D, Werner P and Raine CS (2000) Glutamate excitotoxicity in a model of multiple sclerosis. Nat. Med. 6:67-70

27. Smith ME (1999) Phagocytosis of myelin in demyelinative disease: a review. Neurochem. Res. 24: 261-268

28. Castro-Palomino JC, Simon B, Speer O, Leist M and Schmidt RR (2001) Synthesis of Ganglioside GD3 and Comparison to Bovine GD3 with Regard to Oligodendrocyte Apoptosis and Mitochondrial Damage. Chem. Eur. J. 7:21782184

29. Jung M, Kramer E, Grzenkowski M, Tang K, Blakemore W, Aguzzi A, Khazaie K, Chlichlia K, von Blankenfeld G, Kettenmann H and Trotter J. (1995) Lines of murine oligodendroglial precursor cells immortalized by an activated neu tyrosine kinase show distinct degrees of interaction with axons in vitro and in vivo. Eur. J. Neurosci. 7: 1245-1265

30. Nicholson DW (1999) Caspase structure, proteolytic substrates, and function during apoptotic cell death. Cell Death Differ. 6: 1028-1042

31. Leist M and Jaattela M (2001) Four Deaths and a Funeral: from Caspases to alternative Mechanisms. Mol. Cell. Biol. 2: 589-598

32. Volbracht C, Leist M, Kolb SA and Nicotera P (2001) Apoptosis in caspaseinhibited neurons. Mol. Med. 7: 36-48

33. Ladisch S, Chang F, Li R, Cogen P and Johnson D (1997) Detection of medulloblastoma and astrocytoma-associated ganglioside GD3 in cerebrospinal fluid. Cancer Lett. 120: 71-78

34. Ryu BR, Choi DW, Hartley DM, Costa E, Jou l and Gwag BJ (1999) Attenuation of cortical neuronal apoptosis by gangliosides. J. Pharmacol. Exp. Ther. 290:811816

35. Hicks D, Heidinger V, Mohand-Said S, Sahel J and Dreyfus H (1998) Growth factors and gangliosides as neuroprotective agents in excitotoxicity and ischemia. Gen. Pharmacol. 30: 265-273

36. Koike T, Fehsel K, Zielasek J, Kolb H and Burkart V (1993) Gangliosides protect from TNF alpha-induced apoptosis. Immunol. Lett. 35: 207-212

37. Svennerholm L, Boström K, Jungbjer B and Olsson L (1994) Membrane lipids of adult human brain: lipid composition of frontal and temporal lobe in sujects of age 20 to 100 years. J. Neurochem. 63: $1802-1811$

38. Colell A, Garcia-Ruiz C, Roman J, Ballesta A and Fernandez-Checa JC (2001) Ganglioside GD3 enhances apoptosis by suppressing the nuclear factor-kappa B-dependent survival pathway. FASEB J. 15: 1068-1070

39. Schmied M, Breitschopf H, Gold R, Zischler H, Rothe G, Wekerle H and Lassmann H (1993) Apoptosis of T lymphocytes in experimental autoimmune encephalomyelitis. Evidence for programmed cell death as a mechanism to control inflammation in the brain. Am. J. Pathol. 143: 446-452

40. Stassi G, Todaro M, Bucchieri F, Stoppacciaro A, Farina F, Zummo G, Testi Rand De Maria R (1999) Fas/Fas ligand-driven T cell apoptosis as a consequence of ineffective thyroid immunoprivilege in Hashimoto's thyroiditis. J. Immunol. 162: 263-267

41. Reynolds R and Wilkin GP (1993) Cellular reaction to an acute demyelinating/ remyelinating lesion of the rat brain stem: localisation of GD3 ganglioside immunoreactivity. J. Neurosci. Res. 36: 405-422

42. Heinrich M, Wickel M, Schneider-Brachert W, Sandberg C, Gahr J, Schwandner R, Weber T, Saftig P, Peters C, Brunner J, Kronke M and Schutze S (1999) Cathepsin D targeted by acid sphingomyelinase-derived ceramide. EMBOJ. 18: $5252-5263$

43. Joza N, Susin SA, Daugas E, Stanford WL, Cho SK, Li CY, Sasaki T, Elia AJ, Cheng HY, Ravagnan L, Ferri KF, Zamzami N, Wakeham A, Hakem R, Yoshida $\mathrm{H}$, Kong YY, Mak TW, Zuniga-Pflucker JC, Kroemer G and Penninger JM (2001) Essential role of the mitochondrial apoptosis-inducing factor in programmed cell death. Nature 410: 549-554 
44. Li LY, Luo $X$ and Wang $X$ (2001) Endonuclease $G$ is an apoptotic DNase when released from mitochondria. Nature 412: $95-99$

45. Borner $C$ and Monney L (1999) Apoptosis without caspases: an inefficient molecular guillotine? Cell Death Differ. 6: 497-507

46. Volbracht C, Leist M and Nicotera P (1999) ATP controls neuronal apoptosis triggered by microtubule breakdown or potassium deprivation. Mol. Med. 5 : 477-489

47. Nicotera P, Leist $M$ and Manzo L (1999) Neuronal cell death: a demise with different shapes. Trends Pharmacol. Sci. 20: 46-51

48. Raine CS (1997) The Norton Lecture: a review of the oligodendrocyte in the multiple sclerosis lesion. J. Neuroimmunol. 77: 135-152

49. Bonetti B and Raine CS (1997) Multiple sclerosis: oligodendrocytes display cell death-related molecules in situ but do not undergo apoptosis. Ann. Neurol. 42: $74-84$

50. Schorpp M, JagerR, Schellander K, SchenkelJ, WagnerEF, WeiherH and Angel $P(1996)$ The human ubiquitin $C$ promoter directs high ubiquitous expression of transgenes in mice. Nucleic. Acids. Res. 24: 1787-1788

51. Trotter J, Bitter-Suermann D and Schachner M (1989) Differentiation-regulated loss of the polysialylated embryonic form and expression of the different polypeptides of the neural cell adhesion molecule by cultured oligodendrocytes and myelin. J. Neurosci. Res. 22: 369-383

52. Bottenstein JE and Sato GH (1979) Growth of a rat neuroblastoma cell line in serum-free supplemented medium. Proc. Natl. Acad. Sci. USA 76: 514-517

53. Bansal R, Warrington AE, Gard AL, Ranscht B and Pfeiffer SE (1989) Multiple and novel specificities of monoclonal antibodies 01,04 , and R-mAb used in the analysis of oligodendrocyte development. J. Neurosci. Res. 24: 548-557
54. Knapp PE, Skoff RP and Sprinkle TJ (1988) Differential expression of galactocerebroside, myelin basic protein, and $2^{\prime}, 3^{\prime}$-cyclic nucleotide $3^{\prime}$ phosphohydrolase during development of oligodendrocytes in vitro. J. Neurosci. Res. 21: 249-259

55. Leist M, Fava E, Montecucco C and Nicotera P (1997) Peroxynitrite and NOdonors induce neuronal apoptosis by eliciting autocrine excitotoxicity. Eur. J Neurosci. 9: $1488-1498$

56. Jaattela M, Wissing D, Bauer PA and Li GC (1992) Major heat shock protein hsp70 protects tumor cells from tumor necrosis factor cytotoxicity. EMBO J. 11 : 3507-3512

57. Schierle GS, Leist M, Martinou J-C, Widner H, Nicotera P and Brundin P (1999) Differential effects of Bcl-2 overexpression on fiber outgrowth and survival of embryonic dopaminergic neurons in intracerebral transplants. Eur. J. Neurosci. 11: $3073-3081$

58. Leist M, Single B, Castoldi AF, Kühnle S and Nicotera P (1997) Intracellular ATP concentration: a switch deciding between apoptosis and necrosis. J. Exp. Med. 185: $1481-1486$

59. Leist M, Volbracht C, Kühnle S, Fava E, Ferrando-May E and Nicotera P (1997) Caspase-mediated apoptosis in neuronal excitotoxicity triggered by nitric oxide. Mol. Med. 3: 750-764

60. Graus F, Cordon-Cardo C, Houghton AN, Melamed MR and Old LJ (1984) Distribution of the ganglioside GD3 in the human nervous system detected by R24 mouse monoclonal antibody. Brain Res. 324: 190-194 